I trials of DON was never reached, these findings support the rationale for future clinical trials testing DON and similar drugs against the MYC subgroup of AT/RT as well as other deadly MYC-driven brain tumors.

\section{ATRT-09. CHROMATIN SEGMENTATION ANALYSES IN ATYPICAL TERATOID/RHABDOID TUMORS REVEAL H3K27ME3-MEDIATED EPIGENETIC SILENCING OF TUMOR SUPPRESSOR GENES AND IDENTIFY A NOVEL ROLE OF EZH2, ASSOCIATED WITH ACTIVE CHROMATIN \\ Pascal D. Johann ${ }^{1,3}$, Serap Erkek ${ }^{1,2}$, Lukas Chavez ${ }^{1}$, Martina Finetti ${ }^{5}$, Kornelius Kerl ${ }^{4}$, Marc Zapatka ${ }^{6}$, Amar Gajjar ${ }^{7}$, Martin Hasselblatt ${ }^{8}$, Stefan M. Pfister ${ }^{1,3}$, and Marcel Kool ${ }^{1} ;{ }^{1}$ Division of Pediatric Neurooncology and German Cancer Network, German Cancer Research Center, Heidelberg, Germany, ${ }^{2}$ European Molecular Biology Laboratory, Heidelberg, Germany, ${ }^{3}$ Department of Pediatric Hematology and Oncology, University Hospital, Heidelberg, Germany, ${ }^{4}$ Department of Pediatric Hematology and Oncology, University Hospital, Münster, Germany, ${ }^{5}$ Northern Institute for Cancer Research, Newcastle, UK, ${ }^{6}$ Division of Molecular Genetics, Heidelberg, Germany, ${ }^{7}$ St. Jude Childrens' Research Hospital, Memphis, TN, USA, ${ }^{8}$ Department of Neuropathology, University Hospital, Muenster, Germany.}

Although SMARCB1 mutations represent the only genetic aberrations in atypical teratoid / rhabdoid tumors (ATRT), a comprehensive assessment of the epigenetic effect of SMARCB1 loss has not been achieved so far. We performed ChIP-sequencing for six active and repressive histone marks (H3K4me1, H3K4me3, H3K27me3, H3K27Ac, H3K9me3, H3K36me3) and EZH2 in 11 primary ATRTs, encompassing all three molecular subgroups of ATRT. Chromatin segmentation analyses revealed that across all three subgroups a high percentage of ATRT genomes resides in a quiescent state and active enhancer states are significantly underrepresented as compared to non-neoplastic brain tissues. These data show that loss of SMARCB1 generally results in a subgroup independent repression of genes In addition, we observed gain of the repressive mark H3K27me3 in ATRTs at genes bound by SMARCB1 in normal brain, in line with the known antagonistic role between SMARCB1 and EZH2. Tumor suppressor genes and neuronal transcription factors (such as NEUROD2) were particularly affected by this mechanism of epigenetic silencing. Interestingly, by comparing the genome wide distribution of EZH2 and $\mathrm{H} 3 \mathrm{~K} 27 \mathrm{me} 3$, we also identified classes of ATRT-specific active genes bound by EZH2, but lackingH3K27me3, pointing at a non-canonical role of EZH2 in gene activation. In subgroup ATRT-TYR, which displayed the most striking pattern of EZH2 associated gene activation, this pattern could for instance explain the overexpression of previously identified subgroup specific signature genes such as BMP4. The comprehensive study of histone marks in this genomically simple but epigenetically heterogeneous disease enabled us to not only shed light on the chromatin landscape of these tumors but also on the potentially tumorigenic consequences of SMARCB1 loss. In addition, the dual role of EZH2 in these tumors further enhances the importance of this molecule as a therapeutic target.

ATRT-10. EARLY POST RADIATION CHANGES AND EFFICACY IN CHILDREN WITH ATRT TREATED ON COG ACNS 0333 A COMPARISON OF PROTON VS PHOTON THERAPY Anita Mahajan ${ }^{1}$, Doug Strother ${ }^{2}$, Ian Pollack ${ }^{3}$, Tom Merchant ${ }^{4}$, Chris Williams-Hughes ${ }^{5}$, Allen Buxton ${ }^{5}$, Tianni Zhou ${ }^{5}$, Mark Krailo ${ }^{5}$, and Alyssa Reddy ${ }^{6},{ }^{1}$ Mayo Clinic, Rochester, MN, USA, ${ }^{2}$ Alberta Children's Hospital, Calgary, AB, Canada, ${ }^{3}$ Childrens Hospital of Pittsburgh, Pittsburgh, PA, USA, ${ }^{4}$ Childrens Oncology Group, Arcadia, CA, USA, ${ }^{5}$ St Jude Children's Research Hospital, Memphis, TN, USA, ${ }^{6}$ University of Alabama at Birmingham, Birmingham, AL, USA.

BACKGROUND/OBJECTIVES: COG ACNS0333 is a prospective study evaluating surgery, intensive chemotherapy and radiation therapy (RT) in the management of ATRT. The therapy sequence was based on patient age, disease location and extent. Both proton RT (PRT) and photon RT (XRT) modalities were permitted. In this study the early post-RT changes and efficacy of PRT and XRT were compared. METHODS: All RT plans were reviewed centrally. Pre-operative, post-operative, pre-RT and first post-RT MRI scans were available and reviewed. Patient characteristics, treatment sequence, RT plan, tumor volume, brain and brainstem dosimetric parameters were evaluated. RESULTS: 41 of 65 evaluable patients on protocol received RT between 5/2009 and 11/2013 (14 PRT, 27 XRT). Median age at RT was $1.7 \mathrm{y}$ (range $0.6-13.9 \mathrm{y}$ ). $6 / 41$ patients received craniospinal RT. Median tumor dose was 50.4Gy (range 45-54Gy). All patients had $>1$ postRT MRI scans available. At a median post-diagnosis follow up of 9 months, tumor control was equivalent after PRT and XRT $(\mathrm{p}=0.83)$. At a median post-RT follow up of 5 months (range 1.4-36.1) 10/42 patients had in-field MRI changes of varying severity ( 3 PRT, 7 XRT) in the absence of clear tumor progression. These MRI changes were noted between 2-6 months post-RT. Patients with post-RT MRI changes tended to have larger tumor with larger pre-operative tumor volume (median $137 \mathrm{cc}$ vs $57 \mathrm{cc}$ ) and larger
CTV's (median $257 \mathrm{cc}$ vs $125 \mathrm{cc}$ ); however, the difference was not statistically different $(p=0.21)$. CONCLUSION: In this early analysis of COGACNS 0333 there were no detectable differences in tumor control between the patients treated with PRT vs XRT. Reactive changes were seen with both modalities but were not excessive with either.

\section{ATRT-11. MARKED RESPONSE TO ATEZOLIZUMAB IN A PATIENT WITH RHABDOID TUMOR: A CASE STUDY FROM THE IMATRIX- ATEZOLIZUMAB TRIAL}

Franck Bourdeaut ${ }^{1}$, Meghna Das Thaku ${ }^{2}$, Guillaume Bergthold ${ }^{3}$, and Erin Karski ${ }^{2} ;{ }^{1}$ Institut Curie, Paris, France, ${ }^{2}$ Genentech, Inc., San Francisco, USA, ${ }^{3}$ F. Hoffmann-La Roche Ltd., Basel, Switzerland.

BACKGROUND: Rhabdoid tumors (RTs) are aggressive, rare tumors usually diagnosed in early childhood. They portend poor prognosis, despite multi-modality treatment. Atezolizumab targets programmed death-ligand 1 (PD-L1), leading to enhanced anti-cancer T-cell activity. The iMATRIX-Atezolizumab study (phase I/II, open-label; NCT02541604) assessed safety, pharmacokinetics, and preliminary activity of atezolizumab in pediatric/young adult patients. METHODS: Patients aged $<30$ years with relapsed/refractory pediatric solid tumors received atezolizumab every 3 weeks until loss of clinical benefit $(<18$ years, $15 \mathrm{mg} / \mathrm{kg}$ [maximum dose, $1200 \mathrm{mg}$ ]; $\geq 18$ years, $1200 \mathrm{mg}$ ). RESULTS: In 74 patients for whom data are available (multiple solid tumor types), the pharmacokinetics/safety profile of atezolizumab were consistent with those in adults. One 4-year-old male (palpable malignant RT [left paraspinal soft-parts]) was treated with four doses of atezolizumab over approximately 4 months. The patient experienced one grade 3 treatment-related adverse event (AE; amylase elevation [resolved without treatment or drug interruption]) and one grade $1 \mathrm{AE}$ of special interest (elevated thyroidstimulating hormone). No other grade 3/4 treatment-related AEs were reported. The patient experienced clinical improvement 3 weeks after first atezolizumab infusion with visible regression of the tumor, decreased use of pain medications, increased Lansky performance score (from $60 \%$ to $100 \%$ ) and a return to school. Imaging revealed a $37 \%$ decrease in the target lesion diameter at 6 weeks, and a $32 \%$ decrease at 12 weeks. Following the fourth cycle of treatment, the patient stopped atezolizumab treatment to pursue local control therapy. Biomarker analyses of archival tumor tissue revealed strong PD-L1 immunohistochemistry staining (TC3/ IC2) and a high percentage of CD $8+$ cells in the central tumor $(3.69 \%)$, compared with other enrolled patients (median $0.21 \%$, range 0.0012 $10.28 \%)$. CONCLUSIONS: Encouraging clinical and biomarker results using atezolizumab were observed. Dedicated cohorts for children with either RTs or atypical teratoid RT types have been opened in the ongoing study to further evaluate this signal.

\section{BASIC BIOLOGY}

BIOL-02. INTRACRANIAL TUMORS IN CHILDREN: A 10 YEAR REVIEW FROM A SINGLE TERTIARY HEALTH CARE CENTRE

Quratulain Riaz, Ehsan Naeem, Zehra Fadoo, Maha Dev, and

Naureen Mushtaq; Aga Khan University Hospital, Karachi/Sind, Pakistan.

OBJECTIVE: Brain tumors are the second most common malignancy in children and the most common cause of cancer related deaths. Major advances in management in terms of surgery, radiation and chemotherapy have led to better outcomes. Delayed diagnosis, advanced disease at presentation, late referrals, nosocomial infections, delays to radiotherapy and poor support services are the major reasons for poorer outcomes in developing countries. Little is known about the profile of brain tumors in Pakistan. This study aims to describe the clinical profile and outcome of primary brain tumors in children at a single tertiary center in Pakistan. METHODS/ MATERIALS: All children $(0-16$ years) with primary CNS tumors from 2004 till 2014 at Aga Khan University Hospital were reviewed retrospectively for clinical data, demographics, radiological findings, management and outcome. RESULTS: 175 children were included in the study. Male to female ratio was 1.4:1. Most of the patients were in 5 -10 years age group $(38.9 \%)$. Most common presenting complains were headache $115(65.7 \%)$ \& vomiting $100(57.1 \%)$. Predominant site was supratentorial 91 (52\%). Three patients $(1.7 \%)$ had family history of cancers. Glial tumors were $93(53.1 \%)$ followed by embryonal $40(22.9 \%)$, Craniopharyngiomas 25 $(14.3 \%)$ \& Germ cell $1(0.6 \%)$. Low grade astrocytoma $(23.4 \%)$ were the most common glial tumors while Medulloblastoma $(15.4 \%)$ was the most common embryonal tumor. Majority of the patients underwent surgery only $(53.7 \%)$. Radiation was given to $48(27.4 \%)$ patients. Half of the patients $79(45.1 \%)$ were lost to follow up. Patients expired were $41(23.4 \%), 24$ $(13.7 \%)$ are alive with residual disease while 22 patients $(12.6 \%)$ were cured. CONCLUSION: This is the only study from Pakistan showing 